\title{
Effective disaster recovery using Software defined networking
}

\author{
Thyagaraju, Mrs. Jyothi. K.S, Girish.L \\ PG Student, Associate professor, Assistant Professor \\ Dept of CSE, Cit, Gubbi, Tumkur
}

\begin{abstract}
In this paper we intended to recover server disaster in multiple servers and the disaster such as power outage, link failures, device failures, rerouting of packets, traffic engineering etc, for this problem we proposed a systematic approach for recovering servers from disaster using software-defined-networking schema. SDN separate the control plane and data plane and that control plane is programmable, by doing control plane programmable it increases flexibility of controlling a network infrastructure. SDN provides fast disaster recovery compare to recovery management, SDN requires some method or interface for control plane to communication with data plane .That one mechanism is OpenFlow and for creating virtual networking infrastructure we using mininet tool and for controlling network using controller Opendaylight. We using lispflow mapping for disaster recovery.
\end{abstract}

Keywords - Disaster recovery, Opendaylight controller, OpenFlow, lispflow mapping, map server, DAO, map resolver, data plane

\section{INTRODUCTION}

Now a day's maintain and managing of the network systems are becoming more complex, and also controlling a network by manually becoming more difficult because of increasable amount of growth in network infrastructure, and also recovering disasters is more complex. Because we have to go to that network node, and we have to solve that problem by vender specific solutions, and debug that error, so to overcome this network complexities we go for new technology called software defined networking[1]. SDN is innovative approach to designing, building, and managing network, provides flexibility to control a network.

In this paper we intended to recovery server disaster, and disaster such as power outage, link failures, device failures, rerouting of packets, traffic engineering etc. In this paper we proposed systematic approach for disaster recovery using SDN.

SDN separates the network plane [2], into control plane and data plane, and that control plane is programmable. It increases flexibility, SDN provides fast disaster recovery. SDN requires some method or interface for control plane to communication with data plane. One such mechanism is called as OpenFlow [3] protocols.

In this paper we using opendaylight controller, opendaylight controller is a controller written in java. In this OpenDaylight controller we use LISP flow mapping [4]. LISP is a technology that provides a flexible map and encap framework that can be used overlay network application. For emulating network system we using emulator called Mininet, for creating virtual network

\section{RELATED WORK}

The literature survey related to software defined networking with the use of lisp flow mapping service has been discussed.

The important aspect of software defined networking [1] is to create programmable network that are logically centralized and control the whole network trough laptop, software defined networking schema separates the network planes [2] in to two different planes, and

Fig 1 represents network plane architecture.

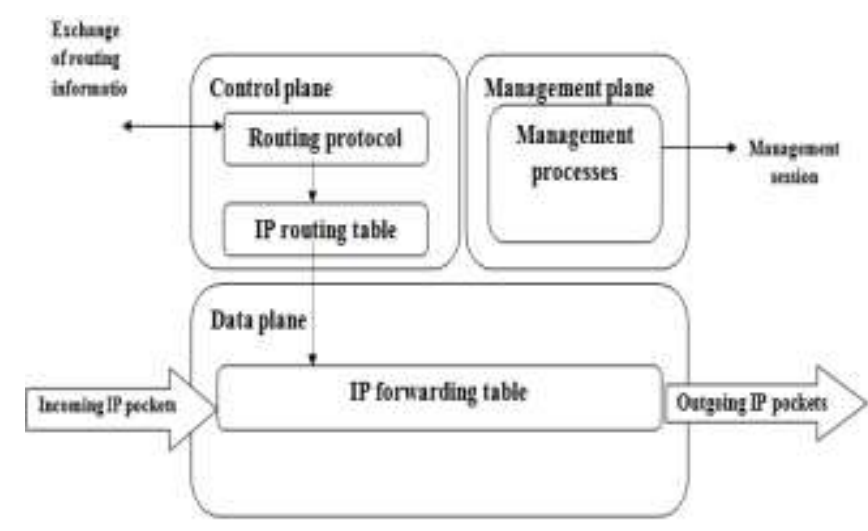

Fig 1: Network planes

The SDN only concentrates about data plane and control plane, data plane contains the physical device like host, switches, and routers.

Data plane is in charge of preparing the travel activity, which chooses what to do with packets landing on an entrance interface. It is likewise termed as sending plane, as it mostly alludes to a sending table to choose legitimate departure interface. From the viewpoint of parcels the Data plane generally handle end-station/client created bundles that are constantly sent by system gadgets to flip side station gadgets.

The control-plane is concerned with collecting, processing and managing the network information in 
order to decide the forwarding protocols that run at control plane. The below are the control plane tasks.

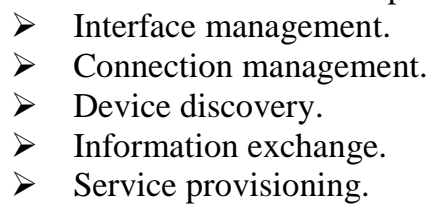

The management plane is used to interact with the network device, in order to manage the network. Management plane also runs its own protocols (such as SNMP), apart from supporting configurations of interfaces, network (IP subnets) and control-plane protocols.

SDN is an approach to building networks that accomplishes the following, provides a global view of network to a centralized controller and Enables external applications to program the network. In SDN, the network automation is logically centralized in software based controllers, Network devices becomes simple packet forwarding devices(data plane) that can be programmed by or via open Interface OpenFlow.

The forwarding hardware is decoupled and it simplifies network management and enables innovation and evolution. SDN proposes the isolation between the control and forward planes from networks, Because of this higher level software can configure the control plane of network packets on demand, enabling the infrastructure to quickly adapt to new application requirements.

\section{A. SDN architecture}

Software Defined Networking [6] is a new technology that proposes the isolation between the control and data plane from the given network. Because of this higher level software to configure the control plane of network packets on demand, priority based packet forwarding enables the infrastructure to quickly adapt new application requirements.

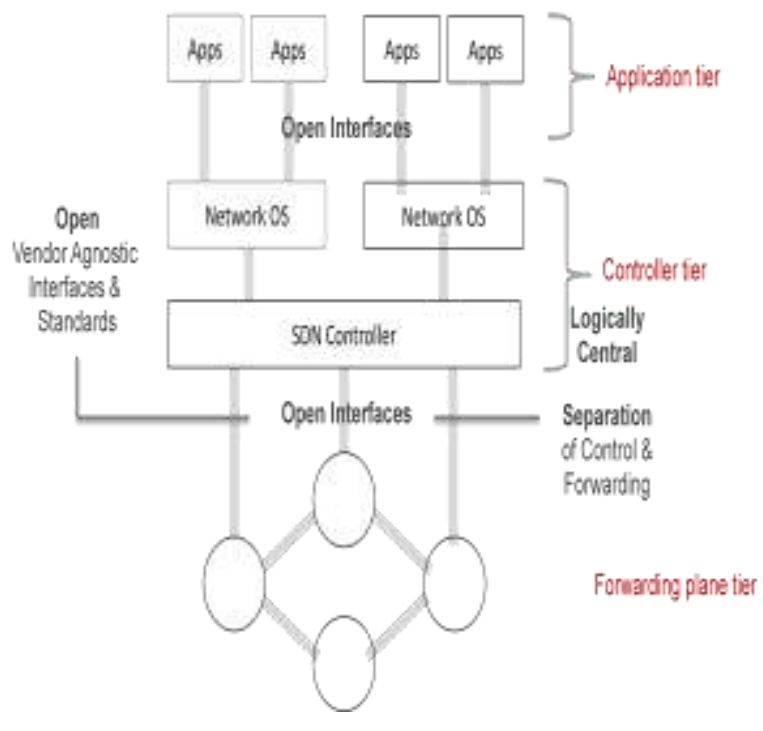

A layered view of this SDN architecture is illustrated in the figure 2.As shown there are three different planes:

Forwarding Plane: It comprises the physical network devices or forwarding devices like switches, routers etc. In SDN such network devices are acting as just forwarding hardware accessible through open interface at an abstraction layer, In SDN such devices are known as switches. In an OpenFlow network, switches [5] are in two types: pure and hybrid. Pure OpenFlow switches have no legacy features and completely rely on controller for forwarding decisions. Hybrid switches support OpenFlow in addition to traditional operation and protocols.

Controller Plane: It includes a logically centralized SDN controller (NOS) that maintains a global view of the network that takes requests through APIs from application plane and performs different operation and monitoring the network devices via standard rules. Network Operating System (NOS) collects physical network state information distributed across every domain and also it empowers the SDN to present a reflection of the physical system state to a case of the control application. The network is programmable through software applications running on top of the NOS that interact with the underlying data plane devices.

Application Plane: Includes arrangements that emphasis on the development of system administrations. These arrangements are predominantly programming applications that correspond with the controller.

Northbound and Southbound APIs: In SDN architecture, northbound application program interfaces (APIs) [7] are used to communicate between the SDN Controller and the applications running on the network. The northbound APIs can be used to provide new application and enable automation of the network to arrange with the needs of different applications via SDN control plane programmability.

Southbound application program interfaces (APIs) provides communication between the SDN Controller and the switches and routers of the network. Southbound APIs provides efficient control over the network and enables the SDN Controller to dynamically make changes according to real-time requirements and demands.

\section{B.OpenFlow}

OpenFlow is an SDN technology that provides a standard way of communication between network devices and controller in SDN architecture. It was proposed to empower analysts to test new thoughts in a generation domain. OpenFlow gives a particular to relocate the control rationale from a switch into the controller. It likewise delivers a convention for the correspondence between the controller and the switches.

Fig 2: SDN architecture 


\section{C. opendaylight SDN controller}

Opendaylight[8] is a SDN controller that is written in java, ODL is a more transparent approach that Fasters the new innovation and reduces risk, opendaylight is a software for forwarding elements , opendaylight controller uses a southbound plug in to interaction between physical devices (data planes), and by using northbound plug-in to expose interface to those writing a applications to the controller, network services and applications to controller, it support application that is run on top of the controller, it support plug-in for inter-controller communication. The figure 3 represents the architecture of ODL controller.

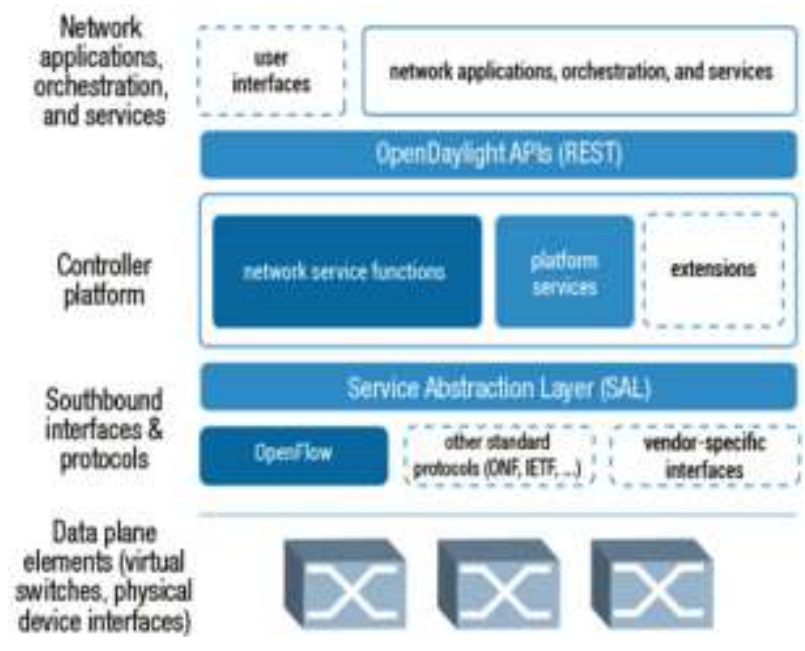

Fig 3: OpendayLight controller architecture

\section{LISP overview}

LISP (location id separation protocol) [4] is a protocol it's try to provide an separation between host identity and host locator and it is done by creating two namespaces. EID (end point identifier) and RLOC (host locator)

LISP provides mapping system between physical IP address to virtual IP address. Where we can create map system between two name spaces EID and RLOC.

\section{E. LISP Flow Mapping Service}

LISP flow mapping service provides mapping system service. LISP flow mapping service contains map server and $\mathrm{mp}$ resolver entities. Mapping data includes mapping of virtual address to physical address. Mapping data can also includes a variety of routing policies includes disaster recovery, traffic engineering and load balancing.

\section{PROPOSED SYSTEM}

Effective disaster recovery using software defined networking in which lisp mobile nodes are installed in client and server nodes for accessing a stream less connectivity between client and server, and
SDN controller uses LISP flow mapping service for disaster recovery in servers.

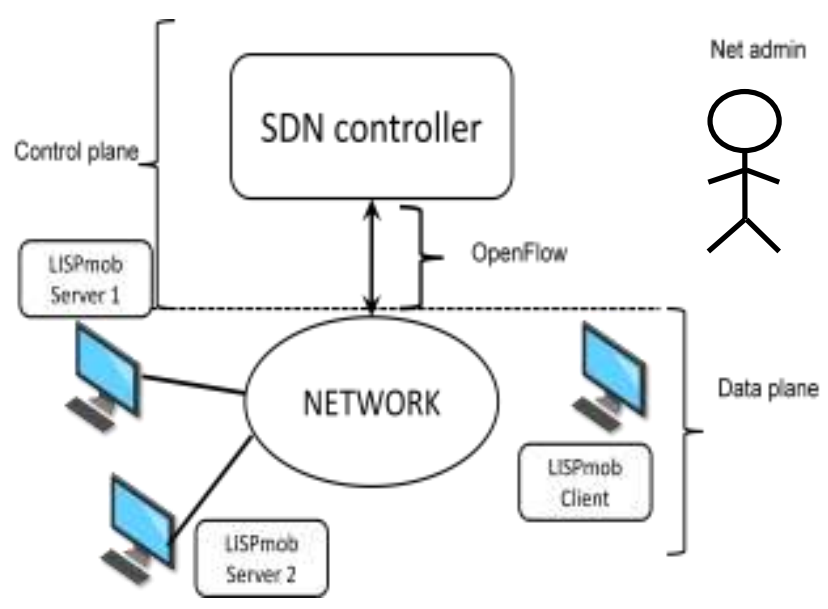

Fig. 4: Architecture of proposed system

Software defined networking (SDN): SDN separates the data and control planes, removes the control plane from network hardware and implemented from software instead, which enables programmatic interface and, it increases flexibility of managing network.

SDN controller: SDN controller is an application in software defined networking (SDN) that manages flow control to enable automation in network management. The controller is the main core of an SDN. It is intermediate between network devices and applications. Any communication between applications and devices has to go through the controller. OpenDaylight is one of the examples of SDN controller and OpenDaylight contains lisp (locator/identifier separation protocol).

Opendaylight: OpenDaylight is an open platform for network programmability to enable SDN and NFV for networks at any size and scale. OpenDaylight software is a combination of components including a fully pluggable controller, interfaces, protocol plug-ins and applications.

Locator/identifier separation protocol (lisp): LISP is a "map-and-encapsulate" protocol which separates the routing locators and identifiers in one number space: the IP address. The advantages of decoupling location and identifier are improved routing scalability, high availability for seamless communication sessions. LISP protocol intern contains map server and map resolver.

Map server: Map server is an open source development environment for building spatially enabled internet applications. Map server which authorizes EID-toRLOC mappings via egress tunnel router (ETR) and adding to the database.

Map resolver: Map resolver which receives map requests from an ingress tunnel router (ITR) and solves the EID to RLOC mapping using a mapping database. Map resolvers used for adding keys to map server and remove key from map server. 
Control plane: The control plane is the layer in a network planes and is responsible for flow control. Functions of the control plane include network configuration and management. In this project we make control plane programmable. That increases flexibility of managing network.

Data plane: The data plane is also called as forwarding plane the part of a network that carries data packets. The data plane is responsible for data transfer between clients, handling multiple conversations using various protocols, and manages communication with remote hosts. Data plane packets travels via routers, rather than to or from the.

Net admin: Responsible for the maintenance of computer hardware and software system that make up a computer network including the maintenance and monitoring of active data network or converged infrastructure.

Lisp mob: LISP mob is an open source application, with LISP mob node can change their network attachment point without losing connectivity between network and host, by maintaining the same IP address.

Lispmobile node: Lisp mobile node typically sends and receives LISP encapsulated packets. It uses the two name spaces endpoint identifier (EID) to name hosts in networks and routing locators (RLOCs) to locate a node.

\section{IMPLEMENTATION}

A. In this paper we divide the implementation into four modules that are given below

$>$ SDN controller configuration.

$>$ Map registering.

$>$ Map server authentication.

$>$ Servers priority checking.

\section{B. Detailed disaster recovery work flow diagram}

In this implementation, the pseudo code for disaster recovery is explained in detail. The detailed project designee flow diagram is as shown in below figure 4 .

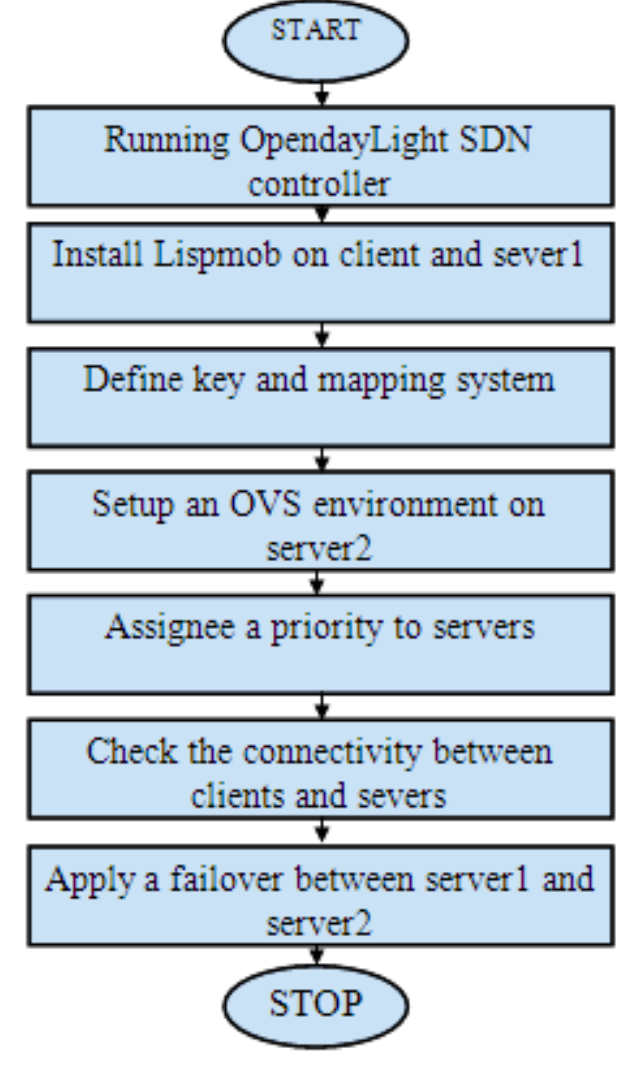

Fig 4: Detailed work flow

Firstly we have to run the SDN controller, in this paper we consider a OpendayLight controller for supporting lisp flow mapping services, and next we have to install the Lisp mob nodes on client and server1 for network configuration.

Next step is to define key and mapping system. Key means the registration and server information are stored in a flow table, this entry is identified by a key, this key is defined by using JSON (java script object notation).

Mapping system main goal is to map the physical address space to virtual address space that is done LISP service.

And next step is to setup an OVS (open virtual switch) environment to create virtual switch to see the fail over between the server 1 and server2.

After creating a virtual switch we have to create bridge between controller and switch after that, assign a priority to server by using a northbound API's and using postman rest API application and check the connectivity between client and server, and see the failover between server1 and serevr2, this can be seen by WireShark interface.

\section{Lisp mapping service implementation}

This below figure represents a lisp flow mapping service implementation internal architecture. 


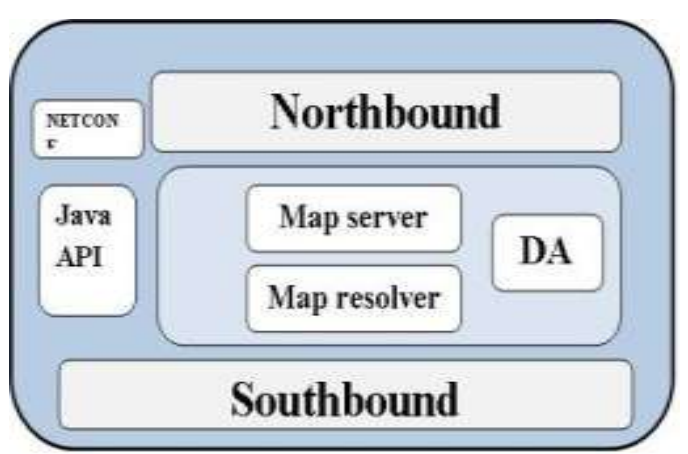

Fig 5: LISP Mapping Service Internal Architecture

DAO (Data access object): it separates a actual database implementation from the user, it creates a isolation between map server and map resolver, this implementation is done by using java API's. The main goal of DAO is to accessing a database without the knowledge of the implementation logic.

Map Server: The main goal of map server is adding and registering a servers and creating a key and mapping system.

Map Resolver: The main job of map resolver is receiving a query and processing queries sending by client and server.

Northbound API's: It is a part of ODL northbound API .This module defining key EID mapping and adding mapping information from map server.

NETCONF: This module activates the LISP service to communication between network active devices trough ODL's plug-in.

Java API's: It is used expose the map server and map resolver tasks through java API.

LISP Southbound plug-in: In order to communicate between with data plane us using LISP southbound plug-in, and in order to support control plane protocol, one of the example for a southbound plug-in is OpenFlow protocol.

\section{D. map registering}

In this module we intended to explain how map registering done and this can by that map server collects the EID and stores in locator array and sends ETR sends map register message to map server for proxy replay and next add EID to locator record to database, if key id matches map register message to map server or else no authentication of data. This can be explained by using below pseudo code and also by using flowchart figure 6 .

\section{1) Pseudo code for map registering:}

Step1: Map server will fetch the EID (End point identifier) and stores in locator list of array.
Step2: ETR requesting a map register message requesting the map server to proxy map replay.

Step3: Map server sends the map notify message to map register.

Step4: Add EID (End point identifier) locator record to database.

Step5: Check for authentication of data.

Step6: If (key_id matches with the map sever key_id).

$$
\text { True: goto (step7); }
$$

False: goto (step8);

Step7: Send the map register / map notify message to map server.

Step8: Send ack to map server to notify message no authentication of data.

2) Flow chart for map registering:

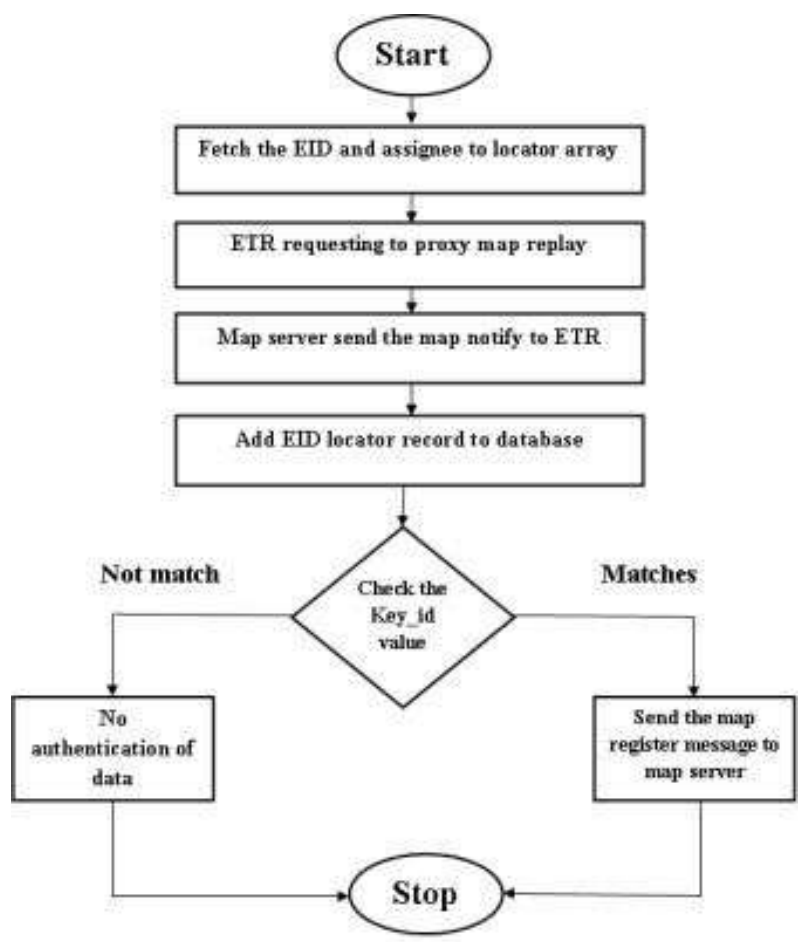

Fig 6: Flow chart for map registering process

\section{E. map server authentication}

In this module we intended explain the how the map server authentication is done. Authentication key is get from map resolver and next map server sends a iterate mask to map resolver. If map resolver sends add key message. Add authentication key to map server otherwise remove authentication key from map server. This can be explained by below pseudo code with flow chart figure 7. 
1) pseudo code for map server authentication:

Step1: Map server should be authenticated.

Step2: Get authentication key from the map resolver.

Step3: Map server sends a iterate mask to map resolver.

Step4: if map resolver send below notify message.

Add key: goto (step5);

Remove key: goto (step 6);

Step5: Add authentication key from map server this method add the authenticate key to map server.

Step6: Remove authentication key from map server.

2) Flow chart for map server authentication

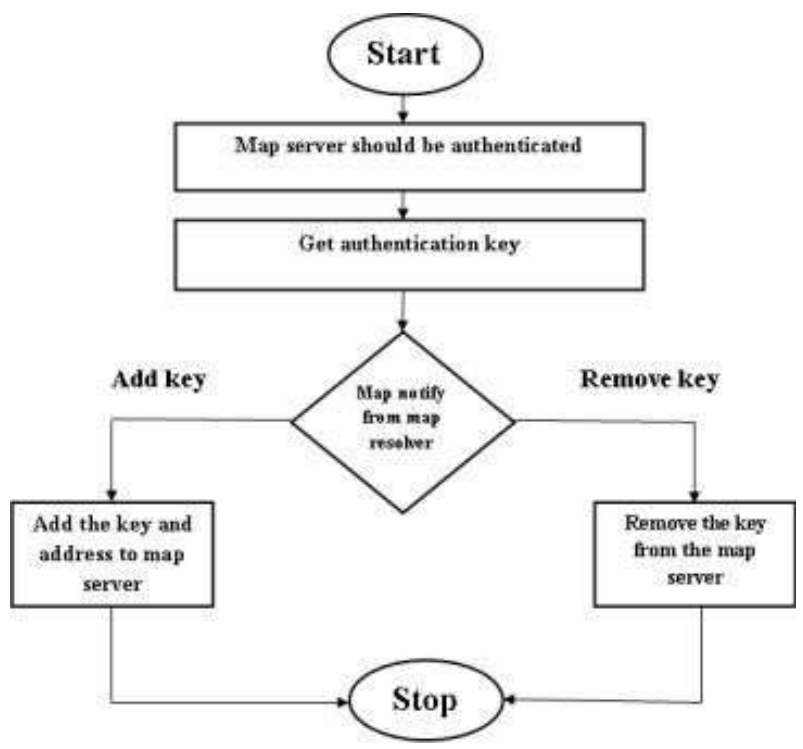

Fig 7: Flow chart for map server authentication

\section{F. Servers priority checking}

In this module we intended to explain checking priorities server by SDN controller. The client sends request to server via controller, the controller checking key_id if key_id matches the controller connects to server for service otherwise controller send notify message called key not matches with map server key_id . the client get service on same time server gets disaster the controller searching for another server with higher priority value in this server2 having higher priority so it's connects to server2 if priority lower so it searching for another server. This can be explained by using below pseudo code with flow chart figure 8 .

\section{1) Pseudo code for servers priority checking}

Step1: Client sends the request to server via SDN controller.

Step2: If (client key_id matches with the map sever key_id).

$$
\text { True: goto (step3); }
$$

False: goto (step4);

Step3: The controller connects to server for services.

Step4: The controllers disconnect the client with notify message called key not matches with map server key.

Step5: server1 crashes or disaster accurse.

Step6: The controller checking for priority by using map resolver.

Step7: If (the priority of server1 < priority of server2).

True: goto (step8);

False: goto (step9);

Step8: migrate to server2.

Step9: checking for another server which having higher priority.

\section{2) Flow chart for priority checking}

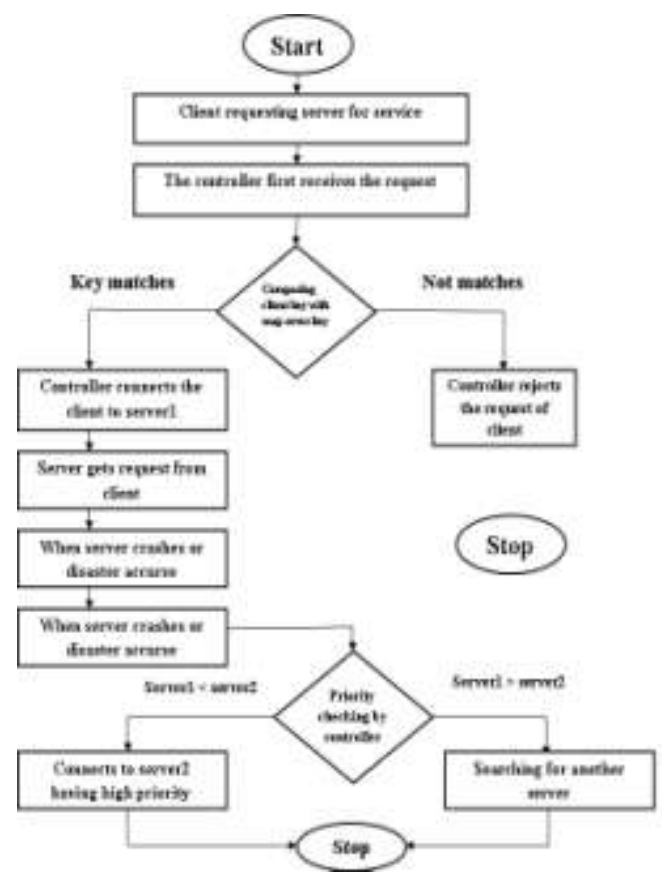

Fig 8: Flow chart for priority checking

In this flow chart figure 8 we intended to explain checking priorities server by SDN controller. The client sends request to server via controller, the controller checking key_id if key_id matches the controller connects to server for service otherwise 
controller send notify message called key not matches with map server key_id . the client get service on same time server gets disaster the controller searching for another server with higher priority value in this server2 having higher priority so it's connects to server2 if priority lower so it searching for another server.

\section{EXPERIMENTAL RESULTS}

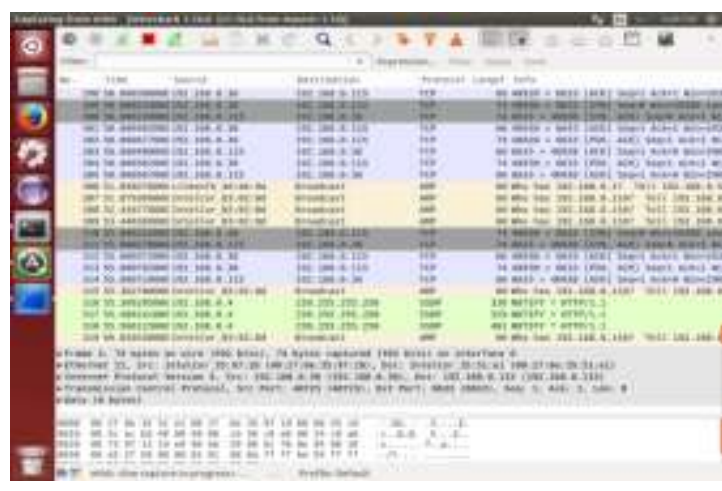

Fig 9: analysing a fail over of server1 to server 2 by using packet analyser wireshark

Result analysis is done by using wireshark packet analyser we can see the fail over between server1 and server2 in figure 9, the SDN controller change priority of server 1 to server 2

\section{CONCLUSION AND FUTURE WORK}

This paper successfully implements the disaster recovery of servers by using LISP flow mapping service and strong concepts of software defined networking over the UBUNTU operating system. We successfully implemented mapping service and priority for servers by using SDN controller and this project future extension is to deploy lisp flow mapping service in windows and android platform.

Future Work: Platform extension: The implementation can be extended to work over android platform.
Downtime reductions: Configurations to reduce the downtime are to be made.

\section{ACKNOWLEDGMENT}

We are grateful to express sincere thanks to our faculties who gave support and special thanks to our department for providing facilities that were offered to us for carrying out this paper

\section{REFERENCES}

[1]https://www.sdncentral.com/resources/sdn/.

[2] Shridar K N Rao," SDN and Its Use Case-NV and NFV" NTIL 2014.

[3] Hao Jin, Deng Pan, Jason Liu, Member and Niki Pissinou IOpenFlow-Based Flow-Level Bandwidth Provisioning for CICQ Switches", IEEE Transactions On Computers, Vol. 62, No. 9, September 2013

[4]https://wiki.opendaylight.org/view/OpenDaylight_Lisp_Flow_Map ping:User_Guide_for__Hydrogen\#Tutorial.

[5] Open Networking Foundation \OpenFlow Switch Speci_cation" Version 1.4.0, October 14, 2013.

[6] Open Networking Foundation ISDN architecture" Issue 1, June 2014.

[7] Diego Kreutz, Fernando M. V. Ramos, Paulo Verissimo, Christian Es-teve Rothenberg, ISoftware-De_ned Networking: A Comprehnsive Sur- vey". Arxiv:1406.044v3 8th October 2014.

[8]http://www.1.4.5.net/ dmm/talks/onug_2013.pdf. 